\section{CRYPTOSPORIDIUM SPP. EM IDOSOS RESIDENTES EM INSTITUIÇÕES DE LONGA PERMANÊNCIA NO SUL DO BRASIL}

\section{CRYPTOSPORIDIUM SPP. IN OLDER ADULTS LIVING IN LONG-TERM CARE FACILITIES IN SOUTHERN BRAZIL}

\author{
Rosiléia Marinho de Quadros ${ }^{1}$, Rafael de Lima Miguel ${ }^{2}$
}

\section{RESUMO}

Introdução: A criptosporidiose é uma doença mundial, levando a quadros desde assintomáticos à diarreia grave. O presente estudo teve como objetivo detectar oocistos de Cryptosporidium spp. em idosos residentes em instituições de longa permanência na cidade de Lages, estado de Santa Catarina, Brasil, buscando avaliar a situação da parasitose, relativamente desconhecida na população idosa, foi avaliada por meio de um método diagnóstico fácil e de baixo custo, porém nem sempre realizado na rotina laboratorial.

Métodos: Foram coletadas amostras dos exames Parasitológicos de fezes de 93 idosos residentes em instituições de longa permanência no período de setembro a novembro de 2015.

Resultados: Os oocistos do protozoário foram detectados pela técnica de Ziehl-Neelsen modificada. A positividade para oocistos de Cryptosporidium spp. foi de 6,45\% (6/93). Em relação ao sexo, a positividade foi de 8,9\% (4/45) para mulheres e 2,1\% (2/48) dos homens. Todos os pacientes tinham idade entre 60 e 70 anos. Em relação à consistência fecal, todas as amostras foram descritas como diarreia.

Conclusão: A criptosporidiose é uma infecção parasitária importante e debilitante que afeta os idosos e precisa ser tratada. Os cuidadores devem estar cientes disso, para que tenham cuidados adequados, especialmente de higiene individual e uso de utensílios que podem transmitir a infecção aos demais idosos que residem nessas instituições.

Palavras-chave: Idosos; fezes; infecção por protozoários; imunocomprometidos

\section{ABSTRACT}

Introduction: Cryptosporidiosis is a worldwide disease whose characteristics range from absence of symptoms to severe diarrhea. The present study aimed to detect Cryptosporidium spp. oocysts in older adults living in long-term care facilities in the city of Lages, state of Santa Catarina, Brazil. The situation of this parasitosis, which is relatively unknown in the older population, was evaluated using an easy and low-cost diagnostic method, although not always performed in laboratory routine.

Methods: Stool samples were obtained from 93 older adults living in long-term care facilities from September to November 2015.

Results: Protozoan oocysts were detected by the modified Ziehl-Neelsen technique. Positivity for Cryptosporidium spp. oocysts was 6.45\% (6/93). Regarding sex, 8.9\% (4/45) of women and $2.1 \%(2 / 48)$ of men were positive. All patients were aged between 60 and 70 years. Regarding fecal consistency, all samples were described as diarrhea.

Conclusion: Cryptosporidiosis is an important, debilitating parasitic infection that affects older age and needs to be addressed. Caregivers must be aware of it in order to provide appropriate care, especially in terms of individual hygiene and use of tools that may transmit infection to other older adults living in these facilities.

Keywords: Aged; feces; protozoan infection; immunocompromised host
Clin Biomed Res. 2018;38(3):223-228

1 Curso de Biomedicina, Laboratório de Parasitologia, Universidade do Planalto Catarinense (UNIPLAC). Lages, SC, Brasil.

2 Curso de Biomedicina, Laboratório de Microbiologia, Universidade do Planalto Catarinense (UNIPLAC). Lages, SC, Brasil.

Autor correspondente Rosiléia Marinho de Quadros biomedvety@gmail.com Universidade do Planalto Catarinense (UNIPLAC)

Av. Castelo Branco, 190. 88509-900, Lages, SC, Brasil. 
O envelhecimento é parte de um procedimento ininterrupto, que termina com o falecimento' ${ }^{1}$. O homem é considerado idoso quando atinge a idade igual ou acima de 60 anos. Atualmente, a população idosa no Brasil está em grande crescimento, contando com 18 milhões de pessoas ${ }^{2}$. Com o aumento desta população, algumas doenças crônicas e degenerativas começam a aparecer em decorrência do processo natural do envelhecimento. A possibilidade de desenvolver doenças, com o avanço da idade, pode ser em virtude do comprometimento do sistema imune ${ }^{3}$.

Entre as doenças oportunistas está a criptosporidiose, uma protozoose nas quais as manifestações clínicas estão diretamente associadas ao comprometimento do sistema imune.

Cryptosporidium é um protozoário pertencente ao filo Apicomplexa, sendo o único representante dentro da família Cryptosporidiidae ${ }^{4}$.

Atualmente 26 espécies de Cryptosporidium são reconhecidas com base em dados morfológicos, biológicos e moleculares, destas quase 20 espécies e genótipos que foram relatados em seres humanos, C. hominis e C. parvum são responsáveis pela maioria das infecções ${ }^{5}$. Ainda sobre as principais espécies de Cryptosporidium importantes na epidemiologia da infecção pelo protozoário fora as citadas anteriormente, existem mais seis espécies como C. hominis, C. meleagridis, C. felis, C. canis, C. suis e C. andersoni ${ }^{6}$. Parece haver diferenças geográficas, sazonais e socioeconômicas na distribuição de Cryptosporidium spp. em seres humanos foram identificados e atribuídos diferenças nas fontes de infecção e nas vias de transmissão ${ }^{7}$.

O ciclo de vida do Cryptosporidium é monoxênico semelhante a outros coccídios entéricos, os quais possuem um ciclo merogônico, com duas gerações de merontes e o ciclo gametogênico que finaliza com a formação de oocistos, estruturas responsáveis pela disseminação e sobrevivência do parasito no ambiente $^{8,9}$.

A criptosporidiose é uma enfermidade de ocorrência mundial, na qual o parasito invade a mucosa intestinal e leva à diminuição das bordas das microvilosidades, reduzindo a absorção de nutrientes e causando sintomas como dores abdominais, desidratação e intensa diarreia, responsável pela eliminação dos oocistos ${ }^{8}$. Geralmente, em pacientes sadios, os sintomas são pouco perceptíveis, porém em indivíduos imunocomprometidos podem causar sintomas como cólicas, flatulência, dor epigástrica, náuseas, vômitos, mal-estar e sinais mais intensos como diarreia aquosa grave (perda de 1 a 20 litros/dia) não sanguinolenta ${ }^{10}$.

As manifestações clínicas da infecção dependem da espécie do parasito, carga parasitária, idade do hospedeiro, estado imunológico, estado nutricional e exposição prévia ao agente; podendo variar de uma forma assintomática a sintomática grave ${ }^{11}$.

Devido à falta de informações em relação às parasitoses em idosos, pode-se atribuir as mesmas condições fisiológicas que ocorrem nas parasitoses em crianças, uma vez que a baixa imunidade e os fatores epidemiológicos podem ser semelhantes para explicar estas infecções ${ }^{12}$.

Como as enteroparasitoses não são consideradas de grande importância, nem notificadas, geralmente são subdiagnosticadas e, assim, apresentam uma carência de dados a respeito da epidemiologia das infecções ${ }^{13}$.

O presente estudo teve como objetivo detectar oocistos de Cryptosporidium spp. em idosos residentes em instituições de longa permanência ("asilos") na cidade de Lages, Santa Catarina, sul do Brasil, buscando avaliar a situação da parasitose pouco conhecida em idosos por meio de um diagnóstico fácil e de baixo custo, porém nem sempre realizado na rotina laboratorial.

\section{MÉTODOS}

Foram coletadas amostras dos exames parasitológicos de fezes de idosos residentes em duas instituições de longa permanência da cidade de Lages, no estado de Santa Catarina, Brasil, no período de setembro a novembro de 2015.

As amostras foram coletadas com o auxílio dos cuidadores dos idosos e acondicionadas em frascos descartáveis (coletor universal) devidamente etiquetados e identificados em relação ao sexo e idade. Foram obtidas informações sobre a presença de alguma enfermidade e o uso de medicamentos. $O$ estudo manteve o sigilo em relação ao nome dos pacientes, bem como informações em relação às medicações.

As amostras foram enviadas sob refrigeração ao Laboratório de Parasitologia e Zoologia da Universidade Planalto Catarinense (UNIPLAC) para a análise no período de 24 horas. Os oocistos do protozoário foram detectados por meio da técnica de coloração de Ziehl-Neelsen modificada e visualizados em microscopia óptica $(1000 \mathrm{x})$. Para a preparação das lâminas, foi realizada a técnica de centrífugo-sedimentação pela formalina-éter (Técnica de Richie) e posterior distensão do esfregaço em lâminas. Em relação à consistência fecal, as amostras foram identificadas como diarreicas e consistentes.

O trabalho foi aprovado pelo Comitê de Ética em Pesquisa em Seres Humanos da Universidade do Planalto Catarinense (UNIPLAC) sob número 1.232.480.

\section{RESULTADOS}


Das 93 amostras de fezes analisadas pelo método de coloração de Ziehl-Neelsen modificada, 48 foram do sexo masculino e 45 do sexo feminino. Em relação às idades, os idosos variaram entre 60 a 90 anos. Os oocistos de Cryptosporidium spp. foram identificados em 6,45\% (6/93), das amostras. A positividade das fezes em relação ao sexo feminino foi de 8,9\% (4/45) e 4,2\% (2/48) para o sexo masculino. Os pacientes estavam na faixa etária entre 60 a 71 anos (Tabela 1).

Em relação à consistência fecal das amostras; $22,32 \%$ (24/93) eram diarreicas e destas $25 \%$ foram positivas para oocistos de Cryptosporidium spp. (Figura 1).

As manifestações clínicas dos pacientes positivos para oocistos de Cryptosporidium spp. foram além da diarreia, desconforto abdominal e mal estar. Os idosos segundo dados das instituições, nenhum deles eram soro reagentes para o vírus HIV a nenhuma internação hospitalar foi realizada em função do quadro clínico, apenas terapia de suporte como dieta alimentar e ingestão de líquidos.

Segundo os responsáveis pelas instituições de longa permanência, os idosos apresentavam algumas enfermidades como doença de Crohn, esquizofrenia e demência; ainda muitos deles eram diabéticos (diabetes mellitus), tinham depressão, hipertensão e

Tabela 1: Sexo e idade dos idosos.

\begin{tabular}{ccc}
\hline \multirow{2}{*}{ IDADES } & \multicolumn{2}{c}{ SEXO } \\
\cline { 2 - 3 } & $\begin{array}{c}\text { Masculino } \\
\text { (amostras } \\
\text { positivas) }\end{array}$ & $\begin{array}{c}\text { Feminino } \\
\text { (amostras } \\
\text { positivas) }\end{array}$ \\
\hline $60-70$ anos & $24(02)$ & $28(03)$ \\
$71-80$ anos & $20(00)$ & $13(01)$ \\
$81-90$ anos & $04(00)$ & $04(00)$ \\
TOTAL & $48(02)$ & $45(04)$ \\
\hline
\end{tabular}



Figura 1: Oocistos de Cryptosporidium spp. (vermelho) em amostras de exames de fezes de idosos corados pela técnica de Ziehl Neelsen (1000 x). alguns com sequelas de acidente vascular cerebral (AVC). Sobre a farmacoterapia administrada aos pacientes, estes usavam medicamentos antiglicemiantes, para hipertensão, ansiolíticos entre outros prescritos em decorrente das patologias individuais ou decorrente da própria idade.

Relacionando a positividade da infecção pelo protozoário, $50 \%$ destes pacientes eram diabéticos e $10 \%$ tinham doença de Crohn, os demais não apresentavam nenhuma relação com outras doenças.

\section{DISCUSSÃO}

Para a OMS até 2025, o Brasil será o sexto país do mundo em número de idosos, porém existe grande desinformação sobre a saúde do idoso, sendo o envelhecimento um dos grandes desafios no futuro para a saúde pública ${ }^{14}$.

A população ao longo do processo natural de envelhecimento ocorre uma redução gradual das capacidades funcionais e fisiológicas dos indivíduos que por consequência aumenta o risco da qualidade de vida do idoso, limitando sua autonomia e aumentando a vulnerabilidade de sua saúde. Segundo dados do Departamento de Informática do Sistema Único de Saúde (DATASUS), no ano de 2014, as doenças infeciosas e parasitárias representaram a sexta causa de morbidade no país ${ }^{15}$.

Os idosos, crianças, gestantes e imunocomprometidos fazem parte da população mais vulnerável às morbidades e complicações quando expostos a microrganismos oportunistas ${ }^{16}$. O número de casos fatais por patógenos entéricos estão entre 10 a 100 vezes maiores em idosos que na população em geral ${ }^{17}$.

Em relação às doenças parasitárias, os idosos apresentam maior predisposição às infecções, sobretudo as oportunistas devido à diminuição das funções do sistema imunológico que ocorrem naturalmente com o processo de envelhecimento, como também associado à presença de enfermidades principalmente crônicas e uso de polimedicamentos que expõem as interações medicamentosas que agridem o sistema imune $^{15}$. Ainda sobre esta afirmação, a diminuição da função imunitária está associada ao processo de envelhecimento conhecido como imunosenescência, que contribui de maneira importante para infecções e inflamações comuns em adultos mais velhos ${ }^{18}$. Diversos estudos epidemiológicos abrangendo a ocorrência de enteroparasitoses são realizados rotineiramente em crianças, porém pouco se sabe em idosos ${ }^{19}$.

Aliado ao comprometimento do sistema imune, grande parte da população idosa apresenta um baixo nível socioeconômico e sofre de alta prevalência de doenças crônicas e infecciosas. Deste modo, a 
pesquisa de doenças em idosos têm grande importância e também fundamental para uma sobrevida desta população ${ }^{20}$.

Embora C. parvum e C. hominis sejam espécies de maior ocorrência no homem, C. parvum tem sido associado às doenças gastrintestinais, podendo até mesmo levar à doença fatal em imunocomprometidos a debilidade severa em imunocompetentes ${ }^{21}$. Em virtude do trabalho não ter sido desenvolvido a nível molecular não se pode afirmar qual espécie do Cryptosporidium spp. a população idosa está acometida, bem como explicar as rotas de transmissão.

A ausência de uma terapia específica no tratamento do Cryptosporidium spp., aliada ao alto número de oocistos excretados $\left(10^{9}\right.$ a $\left.10^{10}\right)$ por indivíduos infectados que nem sempre apresentam infecção clínica, ou ainda pela manifestação de diarreia transitória, faz com que esses pacientes acabem atuando como reservatório da infecção, favorecendo a transmissão e aumentando o potencial de disseminação do protozoário ${ }^{17}$. Sobre o assunto, também se pode ressaltar que os indivíduos infectados e assintomáticos têm grande importância na epidemiologia da criptosporidiose ${ }^{22}$. Em decorrência desta informação, é de suma importância a responsabilidade que os cuidadores de pessoas idosas devem ter na higienização pessoal e no ambiente, a fim de evitar a transmissão do parasito. Diversos fatores podem facilitar o ciclo de infecção, como higiene deficitária, umidade, temperatura e o ambiente ${ }^{23}$. De acordo com as condições fisiopatológicas dos idosos residentes nas casas de longa duração, muitos deles necessitam auxílio para higiene pessoal, uso de fraldas geriátricas e estas condições podem aumentar os riscos de infecção.

Em Teresópolis (RJ), oocistos de Cryptosporidium spp. foram identificados em 16,7\% (17/102) das fezes de idosos através da técnica de Ziehl Neelsen modificada, destes 29,4\% (30/102) apresentaram fezes diarreicas ${ }^{24}$. A presença de diarreia em relação à infecção dos idosos com Cryptospordium spp. foi diretamente proporcional neste estudo na cidade de Lages, onde todas as amostras positivas foram provenientes de idosos com diarreia. Embora a diarreia seja uma das manifestações clínicas mais comuns, nem sempre o protozoário é diagnosticado, uma vez que depende da taxa de excreção de oocistos e a imunidade do infectado, bem como a excreção dos oocistos pode não coincidir com o período sintomático da doença.

A positividade de Cryptosporidium spp. neste estudo em idosos com doença de Crohn não pode ser atribuída a enfermidade, uma vez que a etiologia da doença não é totalmente esclarecida.
A transmissão do Cryptosporidium spp. ocorre principalmente, através da ingestão de oocistos presentes na água ou alimentos. Diante desta situação, locais onde há o confinamento de pessoas como residências de longa permanência, creches, orfanatos e presídios devem ter maiores cuidados em relação à higiene.

A quantidade de oocistos ingeridos parece ter relação com os sintomas, à ingestão de menos de 10 oocistos já pode levar a infecção aos hospedeiros; porém a excreção de $10^{10}$ oocistos/grama de fezes pode ser eliminada em manifestações clínicas da criptosporidiose e assim aumentando as possibilidades de infecção, por isso o cuidado individual e ambiental deve ser rigoroso em condições de aglomeração de pessoas $^{25}$. Ainda cabe ressaltar que uma das principais características biológicas do Cryptosporidium é decorrente da acentuada resistência dos oocistos à desinfecção por cloro, cloramina e outros desinfetantes químicos empregados nos processos de tratamento da água ${ }^{26}$. Em virtude da alta sobrevivência dos oocistos aos processos de desinfecção de rotina na água medidas, profilática de higiene é uma das principais medidas de controle da parasitose.

Embora a técnica de diagnóstico pelo Ziehl Neelsen permita um diagnóstico fácil e rápido para a detecção de oocistos nas fezes, nem sempre este método é o mais sensível, principalmente considerando que pode ocorrer pessoas assintomáticas ou que eliminam poucos oocistos, levando a resultados falsos negativos ou dificultar o diagnóstico ${ }^{27,28}$.

Os exames coproparasitológicos são de fundamental importância para o diagnóstico das enteroparasitoses, e devem ser solicitados na rotina dos serviços de saúde, considerando que as parasitoses intestinais são frequentes na população e, diferentes métodos devem ser usados para evidenciar ovos e larvas de helmintos, assim como cistos e oocistos de protozoários intestinais ${ }^{27}$. Esta afirmação é importante uma vez que a técnica de diagnóstico de oocistos de Cryptosporidium spp. é muito específica e em muitos casos não é solicitado na rotina laboratorial. Assim o diagnóstico da criptosporidiose é baixo ou subestimado na população.

Embora a pesquisa tenha detectado poucos idosos positivos para Cryptosporidium spp., o trabalho teve importância para levantar dados sobre a parasitose que tem relação direta ao sistema imune e poucos casos são registrados em idosos no Brasil, bem como muitos destes idosos com sintomas de diarreia podem ser tratados para outras enfermidades entéricas, tratamentos estes desnecessários uma vez que a criptosporidiose não existe tratamento específico. 
Ainda segundo o estatuto do idoso, as instituições de saúde devem atender aos critérios mínimos para o atendimento às necessidades do idoso, promovendo o treinamento e a capacitação dos profissionais ${ }^{29}$. Faz-se necessário o conhecimento da situação parasitológica de um local pelas autoridades de saúde e a notificação de casos de acordo com critérios epidemiológicos ${ }^{30}$.

A criptosporidiose é uma infecção parasitária importante, que necessita de diagnóstico do protozoário de forma rotineira, bem como ser difundida, principalmente para os cuidadores dos idosos, que devem ter o cuidado, sobretudo na higiene individual dos idosos, bem como também higiene ambiental.

\section{Conflitos de Interesse}

Os autores declaram não ter conflitos de interesse.

\section{REFERÊNCIAS}

1. Moraes EN. Princípios básicos de geriatria e gerontologia. Coopmed. 2009;1:42-7.

2. Brasil. Ministério da Saúde. Estatuto do Idoso. 3. ed. Brasília: Ministério da Saúde; 2013.

3. Stella F, Gobbi S, Corazza ID, Costa RLJ. Depressão no idoso: diagnóstico, tratamento e benefícios da atividade física. Motriz. 2002;8:91-8.

4. Fayer R, Xiao L. Cryptosporidium and Criptosporidiosis. New York: CRC Press; 2008.

5. Ryan U, Fayer R, Xiao L. Cryptosporidium species in humans and animals: current understanding and research needs. Parasitology. 2014;141(13):1667-85. http://dx.doi. org/10.1017/S0031182014001085. PMid:25111501.

6. Cama VA, Bern C, Roberts J, Cabrera L, Sterling CR, Ortega Y, et al. Cryptosporidium Species and Subtypes and Clinical Manifestations in Children, Peru. Emerg Infect Dis. 2008;14(10):1567-74. http:// dx.doi.org/10.3201/eid1410.071273. PMid:18826821.

7. Xiao L. Molecular epidemiology of cryptosporidiosis: an update. Exp Parasitol. 2010;124(1):809. http://dx.doi.org/10.1016/j. exppara.2009.03.018. PMid:19358845.

8. Barta JR, Thompson RCA. What is Cryptosporidium? Reappraising its biology and phylogenetic affinities. Trends Parasitol. 2006;22(10):46868. http://dx.doi.org/10.1016/j. pt.2006.08.001. PMid:16904941.

9. Simone MC, Widme G. Cryptosporidium: parasite and disease. Wien: Springer Science \& Business Media; 2013.
10. Chiuchetta FA. Cryptosporidiosis in a patient with ankylosing spondylitis treated with adalimumab. Rev Bras Reumatol. 2010;50(3):328-32. PMid:21125168.

11. Fayer R, Ungar BLP. Cryptosporidiumspp. and Cryptosporidiosis. Microbiol Rev. 1986;50(4):458-83. PMid:3540573.

12. Hurtado-Guerrero AF, Alencar FH, Hurtado-Guerrero JC. Ocorrência de enteroparasitas na produção de gerontes de Nova Olinda do Norte-Amazonas, Brasil. Acta Amazon. 2005;35(4):487-90. http://dx.doi.org/10.1590/S004459672005000400013.

13. Biscegli TS, Romera J, Candido AB, Santos JM, Candido ECA, Binotto AL. Estado nutricional e prevalência de enteroparasitoses em crianças matriculadas em creche. Rev Paul Pediatr. 2009;27(3):289-95. http://dx.doi.org/10.1590/S010305822009000300009.

14. Organização Mundial da Saúde (OMS). Envelhecimento ativo: uma política de saúde. Brasília: Organização Pan-Americana da Saúde; 2005.

15. Santos PHS, Barros RCS, Gomes KVG, Nery AA, Casotti CA. Prevalência de parasitoses intestinais e fatores associados em idosos. Rev Bras Geriatr Gerontol. 2017;20(2):24453. http://dx.doi.org/10.1590/198122562017020.160137.

16. Organização das Nações Unidas (ONU). Assembléia mundial sobre o envelhecimento: resolução 39/125. Viena: ONU; 1982.

17. Mirzaei M. Prevalence of Cryptosporidium spp. infection in diarrheic and non-diarrheic humans in Iran. Korean $\mathrm{J}$ Parasitol. 2007;45(2):133-7. http://dx.doi. org/10.3347/kjp.2007.45.2.133. PMid:17570977.

18. Monis PT, Thompson RCA. Cryptosporidium and Giardia zoonoses: fact or fiction? Infect Genet Evol. 2003;3(4):233-44. http://dx.doi. org/10.1016/j.meegid.2003.08.003. PMid:14636685.

19. Santos SA, Merlini LS. Prevalência de de Enteroparasitoses na população do município de Maria Helena, Paraná. Cien Saude Colet. 2010;15(3):899905. http://dx.doi.org/10.1590/ S1413-81232010000300033. PMid:20464203.

20. Morais EP, Rodrigues RAP, Gerhardt TE. Os idosos mais velhos no meio rural: realidade de vida e saúde de uma população do interior gaúcho. Texto Contexto Enferm. 2008;17(2):374-83. http://dx.doi.org/10.1590/S010407072008000200021.

21. Varughese EA, Kasper $S$, Anneken EM, Yadav JS. SHP-2 Mediates Cryptosporidium parvum Infectivity in Human Intestinal Epithelial Cells. PLoS One. 2015;10(11):e0142219. http://dx.doi.org/10.1371/journal. pone.0142219. PMid:26556238.

22. McGlade TR, Robertson ID, Elliot AD, Thompson RC. High prevalence of Giardia detected in cats by PCR. Vet Parasitol. 2003;110(3-4):197-205. http://dx.doi.org/10.1016/S03044017(02)00322-9. PMid:12482648.

23. Hunter PR, Hughes $S$, Woodhouse $S$, Syed Q, Verlander NQ, Chalmers RM, et al. Sporadic cryptosporidiosis case-control study with genotyping. Emerg Infect Dis. 2004;10(7):12419. http://dx.doi.org/10.3201/ eid1007.030582. PMid:15324544. 
24. Pereira CRA, Ferreira AP. Ocorrência e fatores de risco da criptosporidiose em felinos de companhia de idosos. Rev Bras Geriatr Gerontol. 2012;15(4):681-91. http://dx.doi.org/10.1590/S180998232012000400009.

25. Karanis P, Kourenti C, Smith $\mathrm{H}$. Waterborne transmission of protozoan parasites: a worldwide review of outbreaks and lessons learnt. $J$ Water Health. 2007;5(1):1-38. http:// dx.doi.org/10.2166/wh.2006.002. PMid:17402277.

26. Franco RMB, Branco N, Leal DAG. Parasitologia ambiental: métodos de concentração e detecção de
Cryptosporidium spp. E Giardia spp. em amostras de água. Rev Patol Trop. 2012;41(2). http://dx.doi.org/10.5216/ rpt.v41i2.19320.

27. Huber F, Bomfim TCB, Gomes RS. Comparação da eficiência da coloração pelo método da safranina a quente e da técnica de centrífugo flutuação na detecção de oocistos de Cryptosporidium em amostras fecais de animais domésticos. Rev Bras Parasitol Vet. 2004;13:81-4.

28. Santos LU, Cantusio R No, Franco RMB, Guimarães JR. Detecção de oocistos de Cryptosporidium spp. e cistos de Giardia spp. em amostras de esgoto bruto ou tratado: avaliação crítica dos métodos. Eng

Sanit Ambient. 2011;16(2):115-20.

http://dx.doi.org/10.1590/S1413-

41522011000200003.

29. Brasil. Ministério da Saúde: estatuto do idoso. 2. ed. Brasília: Ministério da Saúde; 2007.

30. Merigui EAG, Felippe HP, Badaró RG, Souza LO, Fernandes FM. Avaliação da incidência de parasitoses em uma população idosa em abrigo público, na cidade de Eugenópolis (MG). Rev Cient Faminas. 2011;7:25-36.

Recebido: 31 maio, 2018 Aceito: 27 ago, 2018 